\title{
Late pulmonary sequelae after childhood bone marrow transplantation
}

Isa Cerveri, Maria C Zoia, Paola Fulgoni, Angelo Corsico, Lucio Casali, Carmine Tinelli, Marco Zecca, Giovanna Giorgiani, Franco Locatelli
Recent advances in transplant immunobiology, supportive care, and prevention of graft versus host disease (GVHD) have increased the success of bone marrow transplantation (BMT). Considering the long life expectancy of bone marrow transplant recipients, particularly children, attention is now being focused on the late sequelae of treatment on organ system function.

Pulmonary complications are a major cause of morbidity and mortality in the early period after BMT. ${ }^{1}$ However, there is little published information on long term respiratory abnormalities in transplanted children and available data are somewhat conflicting. ${ }^{2-7}$ The variation between studies may result from the different underlying diseases, different types of transplantation, and the change in therapeutic strategies over time. Furthermore, there is uncertainty over the relationship between changes in lung function and the malignancy, the effects of toxicity of the drugs used to induce remission of the disease, those of the preparative regimens, type of transplant, and transplant related complications.

We report here the results of a cross sectional study carried out on a group of young patients who were given autologous or allogeneic bone marrow transplants during childhood for haematological malignancies.

University of Pavia,

Italy

I Cerveri

M C Zoia

P Fulgoni

A Corsico

L Casali

Unit of Biometrics, IRCCS "S. Matteo", Pavia, Italy

C Tinelli

Department of

Paediatrics, IRCCS

Policlinico "S.

Matteo", University of

Pavia, Italy

M Zecca

G Giorgiani

F Locatelli

Correspondence to: Dr I Cerveri, Istituto Forlanini, Universita' di Pavia, IRCCS S. Matteo, Via Taramelli, 5-27100 Pavia, Italy.

Received 6 April 1998 Returned to author 1 September 1998 Revised manuscript received

5 October 1998

Accepted for publication

5 November 1998 age of patients with lung function abnormalities was higher in those who (1) received a bone marrow transplant after two or three complete remissions compared with those who were transplanted immediately after the first remission (54\% vs $21 \%$; $<0.02$ ), (2) underwent allogeneic bone marrow transplantation rather than an autologous transplantation $(45 \%$ vs $26 \%$; $p=0.06$ ), and (3) had a pulmonary infection compared with those without ( $56 \%$ vs $26 \%$; $p=0.07$ ).

Conclusions-In spite of the absence of chronic respiratory symptoms there is a high prevalence of children with late pulmonary sequelae after bone marrow transplantation. Regular testing is recommended after transplantation, in particular in subjects at higher risk of lung injuries, such as those receiving transplants after more than one remission, those receiving allogeneic transplants, and those having suffered from pulmonary infections. When lung function abnormalities become apparent, long term follow up is necessary to see whether they become clinically relevant. All patients should remain non-smokers after transplantation and should have active early and aggressive treatment for respiratory illnesses.

(Thorax 1999;54:131-135)

Keywords: bone marrow transplantation; lung function

\section{Methods}

PATIENT SELECTION AND STUDY PROTOCOL Between 1986 and 1994, 134 children with haematological malignancies underwent BMT in our Pediatric Department; 52 of the 71 survivors were included in this cross sectional study, 10 were living abroad and therefore could not be evaluated, and nine were not able to cooperate in performing lung function tests because they were under six years old. All patients were examined at least three years after transplantation (range 3-11 years). The characteristics of the population studied are reported in table 1 .

All information about the original malignant disease, first line treatment, conditioning regimen, type of BMT, donor, GVHD prophylaxis, and any early or late complications including respiratory symptoms and diseases were obtained from clinical records. In addition, a detailed history of smoking habit, respiratory symptoms, and diseases was recorded directly from the patients with the aid of their parents. A complete clinical physical examination and lung function tests were then performed in all patients. 
Table 1 Characteristics of patients included in the study

Male/female ratio

Mean age (range) at cross sectional observation (years)

Mean age (range) at BMT (years)

Mean interval (range) between BMT and cross sectional observation (years)

Number of patients transplanted up to December 1990

Number of patients transplanted from 1991 to 1994
CLINICAL DATA COLLECTION

Twenty five patients had suffered from acute lymphoblastic leukaemia (ALL), 22 acute myelogeneous leukaemia (AML), and five chronic myelogeneous leukaemia (CML). Patients with AML were treated according to different cooperative protocols of the Italian Association of Paediatric Haematology/ Oncology (AIEOP). ${ }^{8}$ In particular, all children received an identical induction therapy including daunorubicin (DNR) and cytosine arabinoside (Ara-C) according to the classical scheme $3+7$, followed by a second course of two days DNR and five days Ara-C. One or two courses of consolidation therapy with high dose Ara-C, etoposide, and mitoxantrone were administered before autologous or allogeneic BMT.

Children with ALL received different AIEOP first line protocols-namely, 8503 or 8703 , which were based on the experience of studies like those of the Children Cancer Group, and 8803 and 9103 which were based on the Berlin-Frankfurt-Munster stratification and treatment criteria. $^{10-12}$ These protocols included multiple cytotoxic drugs such as prednisone, vincristine, DNR, low and high dose methotrexate, Ara-C, L-asparagimase, and 6-mercaptopurine. Details of the above two year front line treatments are reported elsewhere. $^{10-12}$

Bone marrow transplantation was performed in 23 patients during their first complete haematological remission and in 24 patients during their second or third remissions following medullary relapses. Patients with CML were considered to be comparable to patients given BMT during the first remission on the basis of the usually mild previous chemotherapy received.

Nineteen patients were given autologous bone marrow transplants and 33 received allogeneic transplants.

Thirty nine patients had received a conditioning regimen including fractioned total body irradiation (12 Gy divided into six fractions over three days), 11 had received a conditioning regimen including busulphan, and two a regimen of BCNU, amsacrine, Ara-C, and VP16.

After BMT 25 children developed acute GVHD (grade I-III) and eight developed chronic GVHD. Acute GVHD was classified according to previously described criteria ${ }^{13}$ and was treated with steroids as first line therapy and horse antilymphocyte globulin in resistant cases. Chronic GVHD was diagnosed according to the criteria published by Shulman ${ }^{14}$ and treated with cyclosporin A and steroids when necessary.

Thirty of the 33 patients given an allogeneic transplant had a positive serological test for human cytomegalovirus (HCMV) prior to BMT. Seven patients had HCMV reactivation. Eight patients developed at least one episode of pulmonary infection: one had two episodes (one about three years after BMT), one had one episode of pneumonia and subsequently two episodes of acute bronchitis, one had four episodes of pneumonia (the last two years after BMT), and one developed pulmonary aspergillosis. Acute bronchitis was defined as an acute episode of airway inflammation with cough and mucopurulent phlegm requiring treatment; pneumonia was defined as the occurrence of an acute pulmonary infection with physical and radiographic signs of parenchymal shadowing. Isolation of bacterial, mycotic and viral agents from secretions obtained by deep coughing was attempted.

The clinical features of the patients included in this study are summarised in table 2 .

\section{RESPIRATORY SYMPTOMS}

The presence of cough and/or phlegm, dyspnoea, and wheezing both at rest and during exercise was recorded at follow up appointments, the details coming directly from the patients or from their parents. Information on the entire period following transplantation was obtained from clinical records. Patients had been routinely visited every three months after BMT.

\section{PULMONARY FUNCTION TESTS}

Measurements of lung volumes were obtained using a water sealed spirometer (Pulmonet III, Sensor Medics, Anaheim, California, USA) and were performed according to the European Community for Coal and Steel (ECCS) statements ${ }^{15}$ and the American Thoracic Society (ATS) recommendations. ${ }^{16}$ The best of three forced vital capacity (FVC) measurements was recorded as well as forced expiratory volume in one second $\left(\mathrm{FEV}_{1}\right), \mathrm{FEV}_{1} / \mathrm{FVC}$ ratio, and maximal expiratory flow at $25 \%$ of FVC $\left(\mathrm{MEF}_{25}\right)$.

Transfer factor for carbon monoxide (TLCO) was determined using the single breath method (Transferscreen-II Jaeger, Wuerzburg,

Table 2 Clinical features of patients included in the study

$\begin{array}{lr}\text { Number of patients receiving BMT by number of } \\ \text { remissions and disease: } \\ \text { In first complete remission } \\ \text { ALL } \\ \text { AML } & 9 \\ \text { CML } & 5 \\ \text { In second and third complete remission } & 16 \\ \text { ALL } & 8 \\ \text { AML } & \\ \text { Number of patients by number of remissions and type } & \\ \text { of transplant: } & 10 \\ \text { In first complete remission } & 18 \\ \text { Autologous BMT } & 9 \\ \text { Allogeneic BMT } & 15 \\ \text { In second and third complete remission } & \\ \text { Autologous BMT } & 25 \\ \text { Allogeneic BMT } & 8 \\ \text { Number of patients with GVHD: } & 7 \\ \text { Acute } & 8 \\ \text { Chronic } & \\ \text { Number of patients with HCMV reactivation } & \\ \text { Number of patients with pulmonary infection } & \end{array}$

$\mathrm{BMT}=$ bone marrow transplantation; $\mathrm{ALL}=$ acute lymphoblastic leukaemia; $\mathrm{AML}=$ acute myelogeneous leukaemia $\mathrm{CML}=$ chronic myelogeneous leukaemia $; \mathrm{GVHD}=$ graft versus host disease; HCMV = human cytomegalovirus. 
Table 3 Mean (SD) values of lung function parameters and percentages of patients with negative $S D$ scores and clearly pathological SD scores

\begin{tabular}{llll}
\hline & $\begin{array}{l}\text { Mean }(S D) \\
\text { value }\end{array}$ & $\begin{array}{l}\text { \% of patients with negative } \\
\text { values }(0 \text { to }>-1.64)\end{array}$ & $\begin{array}{l}\text { \% of patients with pathological } \\
\text { values }(\leqslant-1.64)\end{array}$ \\
\hline FVC & $-0.73(1.40)$ & 52 & 23 \\
TLCO & $-0.92(1.79)$ & 48 & 28 \\
FEV $_{1}$ & $-0.29(1.45)$ & 60 & 10 \\
FEV $_{1} /$ FVC & $0.39(1.05)$ & 35 & 0
\end{tabular}

$\overline{\mathrm{FVC}}=$ forced vital capacity; TLCO $=$ carbon monoxide transfer factor; $\mathrm{FEV}_{1}=$ forced expiratory volume in one second.

Germany) and corrected for haemoglobin content. Since the correction of TLCO for alveolar volume did not influence the results of our analysis, only uncorrected TLCO values are reported. Measurements were performed according to the $\mathrm{ECCS}^{15}$ and $\mathrm{ATS}^{17}$ guidelines. Since this test is more difficult and requires greater cooperation to perform than FVC, TLCo data are unavailable for six patients.

Functional data were expressed as an SD score (actual result-predicted values/ population standard deviation) and defined as pathological when less than -1.64 corresponding to less than the 5 th percentile. Taking into account the pubertal stage of each subject evaluated according to Tanner's method, the SD score was corrected according to the tables reported by Rosenthal et al. ${ }^{18}$ Reference values used were those from a recent cross sectional study on lung function in healthy schoolchildren aged $4-19$ years. ${ }^{19} 20$

Three patterns of respiratory function abnormalities were defined as follows: (1) restrictive (FVC SD score less than -1.64 with $\mathrm{FEV}_{1} /$ FVC SD score ratio more than -1.64); (2) obstructive $\left(\mathrm{FEV}_{1} \mathrm{SD}\right.$ score less than -1.64 with $\mathrm{FEV}_{1} / \mathrm{FVC}$ SD score ratio equal to or less than -1.64); (3) isolated diffusing impairment: (TLCo SD score less than -1.64 and other parameters in the normal range).

STATISTICAL ANALYSIS

$\chi^{2}$ tests for independent samples were applied to verify the null hypothesis of independence between the lung function abnormalities and a series of clinical and treatment parameters. As dependent variables we considered the pathological respiratory function, grouping all the three above mentioned patterns versus the normal one. As independent variables we considered disease status at the time of BMT, subdividing children into those given transplantation during the first remission and those

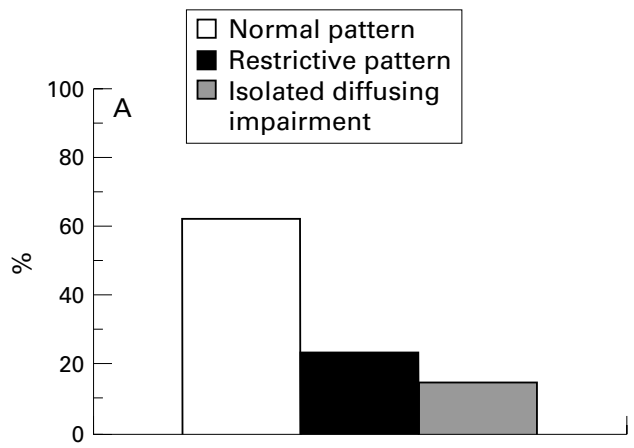

transplanted in more advanced disease, age at BMT, period of time in which BMT was performed (before or after 1990), time interval between BMT and cross sectional observation, type of BMT, inclusion of total body irradiation (TBI) in the conditioning regimen, presence of acute and/or chronic GVHD, reactivation of HCMV infection, and the presence of pulmonary infections.

\section{Results}

At examination patients were found to be free of acute rejection, infection, active GVHD, or any other acute disease. None of the patients smoked and no patient reported chronic respiratory symptoms. The young subjects who were unable to collaborate with the lung function testing did not have any chronic respiratory symptoms according to their parents.

Table 3 reports the mean (SD) values of the functional parameters and percentages of patients with SD scores still in the normal range but negative (between 0 and -1.64) and of those with clearly pathological SD scores (less than -1.64). The mean values of all the functional parameters were still in the normal range although $\mathrm{FVC}, \mathrm{FEV}_{1}$ and, in particular, TLCO were negative; in fact, there was a significant proportion of patients with negative and clearly pathological FVC and TLCo values.

The distribution of respiratory function patterns showed that only $62 \%$ of patients had normal respiratory function. No patient had an obstructive pattern, $23 \%$ had a restrictive pattern of whom $44 \%$ also had a pathological TLCO, and a substantial group of patients (15\%) had an isolated impairment of TLCO with normal lung volumes. When patients were grouped according to the type of BMT, the percentage with a normal pattern greatly increased in the group that had received an autologous BMT (74\%). In fact, no patient in this group had an isolated impairment of TLCO (fig 1).

As far as the associations between the different variables and lung function abnormalities are concerned, the status of the underlying disease at the time of BMT was found to have a statistically significant effect $(\mathrm{p}<0.02)$. The percentage of patients with lung function abnormalities was significantly higher in the patients who received a bone marrow transplant after two or three complete remissions compared with those who were transplanted immediately after

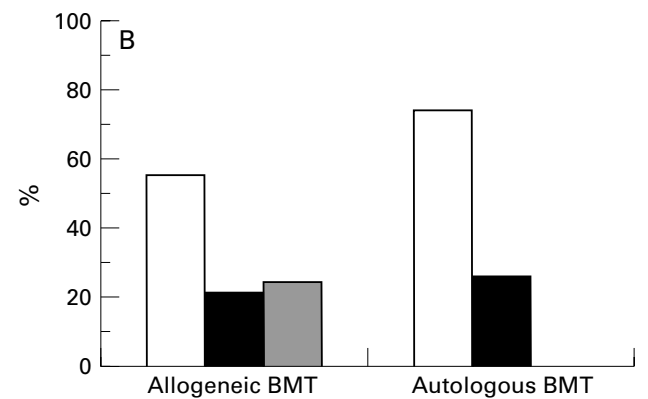

Figure 1 Distribution of respiratory function patterns in $(A)$ all the study patients and $(B)$ in those receiving allogeneic and autologous bone marrow transplantation (BMT). 


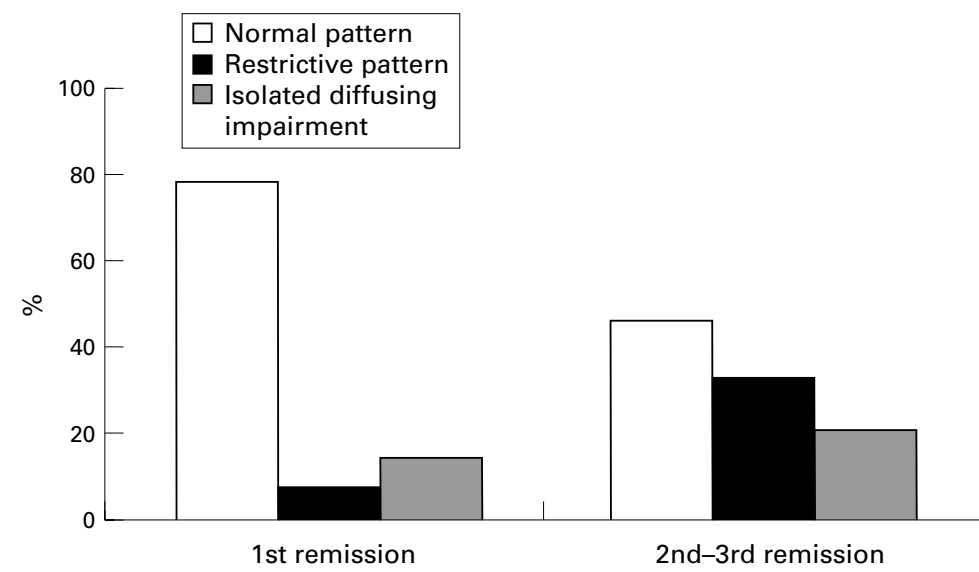

Figure 2 Distribution of respiratory function patterns in patients receiving bone marrow transplantation (BMT) during first remission and in those transplanted after the second or third remission. had been heavily retreated with conventional chemotherapy, surgery, and radiotherapy before receiving the conditioning procedure. Moreover, the sample of surviving patients was biased by the very poor prognosis of these diseases (53 patients alive vs 350 transplanted). An interesting common result was, however, the scarcity of respiratory symptoms even in advanced disease.

Our large sample size allowed us to examine the effect of several potential risk factors, including type of transplant, on lung function abnormalities. We were able to establish that to receive BMT during the second or third remission would require a larger amount of chemotherapy as first line treatment, and this appears to be the most significant determinant of more permanent lung injury. In contrast, receiving less chemotherapy, as in patients with CML or those receiving BMT after a first remission, appears to be a significant factor in preserving lung function. These results support our previous conclusions ${ }^{7}$ on the correlation of toxicity with first line therapy. Unfortunately the number of children receiving BMT for nonmalignant disorders in our cohort of patients was too small to allow us comparisons that would have provided a more definitive statement. In addition, there appear to be different individual susceptibilities to drug toxicity that precludes a precise identification of the subjects at greater risk.

Allogeneic BMT with consequent immunosuppression and more frequent pulmonary infections can contribute to impairment of lung function. A possible explanation for the higher incidence of lung function abnormalities observed in recipients of allogeneic bone marrow transplants compared with children given autologous transplants could be immune mediated lung damage caused by donor lymphocytes.

Both busulphan and TBI in the conditioning regimen are known to cause lung toxicity; we did not find any significant difference between them, unlike Beinert et $a l^{21}$ who found a significantly higher incidence of long term lung impairment with busulphan conditioning than with TBI; it is possible that our data are biased by the non-homogeneous distribution of the type of conditioning in our sample.

Unfortunately our study is cross sectional and lacks baseline respiratory function data. However, given the young age at which the patients developed their disease, it is unlikely that respiratory function was already abnormal. There is one longitudinal study with a long term follow up ${ }^{6}$ but this, too, lacks pulmonary function tests before transplantation for most of the patients. The homogeneous population sample (all patients had received allogeneic transplants) and the relatively small number of patients did not allow all risk factors to be evaluated.

We conclude that, in many patients, lung function abnormalities are present many years after childhood BMT for haematological malignancies even in the absence of chronic respiratory symptoms. After BMT regular monitoring of lung function in all subjects is recommended and, in particular, in those at 
higher risk of lung injuries such as those with more than one remission, those receiving allogeneic BMT, and those having suffered from pulmonary infections. If lung function abnormalities become apparent, long term follow up is necessary in order to see whether they become clinically relevant. As recipients of bone marrow transplants have a long life expectancy and may have to cope with risk factors such as smoke, pollution, infections and ageing in addition to their possible limited pulmonary function reserve, they should remain non-smokers and have early and aggressive treatment for respiratory illness, perhaps including pneumococcal vaccination.

Grant support: Current Research Project, IRCCS, Policlinico S. Matteo, Pavia, No. 681RCR96/02.

1 Breuer R, Iossos IS, Berkman N, et al. Pulmonary complications of bone marrow transplantation. Respir Med 1993;87:571-9.

2 Quigley PM, Yeager AM, Loughlin GM. The effects of bone marrow transplantation on pulmonary function in children. Pediatr Pulmonol 1994;18:361-7.

3 Kaplan EB, Wodell RA, Wilmott RW, et al. Late effects of bone marrow transplantation on pulmonary function in children. Bone Marrow Transplant 1994;14:613-21.

4 Arvidson J, Bratteby LE, Carlson K, et al. Pulmonary function after autologous bone marrow transplantation in chiltren. Bone Marrow Transplant 1994;14:117-23.

5 Nenadov Beck M, Meresse V, Hartmann O, et al. Long-term pulmonary sequelae after autologous bone marrow transpulmonary sequelae after autologous bone marrow transplantation in children without total

6 Nysom K, Holm K, Hesse B, et al. Lung function after allogeneic bone marrow transplantation for leukaemia or lymphoma. Arch Dis Child 1996;74:432-6.

7 Fanfulla F, Locatelli F, Zoia MC, et al. Pulmonary complications and respiratory function changes after bone marrow transplantation in children. Eur Respir $\mathcal{F} 1997 ; 10$ 2301-6.
8 Amadori S, Ceci A, Comelli A, et al. Treatment of acute myelogenous leukemia in children: results of the Italian co-operative study AIEOP/LAM 8204. F Clin Oncol 1987; 5:1356-63.

9 Arcese V, Amadori S, Testi AM, et al. Allogenic vs autologous BMT vs intensive chemotherapy in childhood ALL during first complete remission: AIEOP experience. Bone Marrow Transplant 1991;7(Suppl.3):71-4.

10 Conter V, Aricò M, Valsecchi MG, et al. Extended intrathecal methotrexate may replace cranial irradiation for
prevention of CNS relapse in children with intermediate prevention of CNS relapse in children with intermediate
risk acute lymphoblastic leukaemia treated with BerlinFrankfurt-Munster based intensive chemotherapy. $f \mathrm{Clin}$ Oncol 1995;13:2497-502.

11 Reiter A, Schrappe M, Wolf-Dieter L, et al. Chemotherapy in 998 unselected childhood acute lymphoblastic leukaemia patients: results and conclusions of the multicenter trial ALL-BFM 86. Blood 1994;84:3122-33.

12 Paolucci G, Masera G, Vecchi V, et al. Treating childhood acute lymphoblastic leukaemia (ALL): summary of ten years experience in Italy. ALL Steering Committee of the Associazione Italiana Ematologia Oncologia Pediatrica (AIEOP). Med Pediatr Oncol 1989;17:83-91.

13 Glucksberg H, Storb R, Fefer A, et al. Clinical manifestations of graft-versus-host disease in human recipients of marrow from HLA-matched sibling donors. Transplantation marrow from HLA

14 Shulman HM, Sullivan KM, Weiden PL, et al. Chronic graft-versus-host syndrome in man. Am f Med 1980;69: 204-17.

15 Report of Working Party on "Standardization of lung function tests". Eur Respir f 1993;6:S16.

16 American Thoracic Society. Standardization of spirometry. Am 7 Respir Crit Care Med 1995;152:1107-36.

17 American Thoracic Society. Single-breath carbon monoxide diffusing capacity (transfer factor). Am $\mathcal{F}$ Respir Crit Care Med 1995;152:2185-98.

18 Tanner JM. Growth at adolescence. 2nd ed. Oxford: Blackwell, 1962.

19 Rosenthal M, Bain SH, Cramer D, et al. Lung function in white children aged 4 to 19 years: I. Spirometry. Thorax 1993;48:794-802.

20 Rosenthal M, Cramer D, Bain SH, et al. Lung function in white children aged 4 to 19 years: II. Single breath analysis and plethysmography. Thorax 1993;48:803-8.

21 Beinert T, Dull T, Wolf K, et al. Late pulmonary impairment following allogeneic bone marrow transplantation. Eur $\mathcal{F}$ Med Res 1996;1:343-8. 